\title{
Diagnóstico e proposta de descrição metodológica para artigos técnico- científicos que tratam da avaliação de aplicações de herbicidas ${ }^{1}$
}

\author{
Diagnosis and methodological description proposal for scientific-technological \\ articles relational to assessment of herbicides application
}

\author{
Marcelo Gonçalves Balan²; Otavio Jorge Grigoli Abi Saab ${ }^{3}$; Cleber Daniel de Goes Maciel ${ }^{2}$; \\ Gustavo Migliorini de Oliveira ${ }^{4}$
}

Resumo - O controle químico é uma ferramenta viável e praticamente indispensável no manejo de plantas daninhas. No entanto, detalhes insuficientes sobre os métodos de aplicação de produtos fitossanitários têm sido observados na maioria dos artigos científicos que tratam da utilização de herbicidas. Com objetivo de verificar a presença ou ausência de informações básicas sobre a aplicação de herbicidas encontradas em artigos científicos, assim como propor um critério de descrição mínima necessária de informações que garanta melhor repetibilidade dos métodos e tecnologias utilizadas, foi realizado levantamento em 200 trabalhos publicados em periódicos nacionais e internacionais. Observou-se que descrições mínimas requeridas para tecnologia de aplicação de herbicidas não tem sido atendidas. Apesar de todos os trabalhos apresentarem a taxa de aplicação utilizada $\left(\mathrm{L} \mathrm{ha}^{-1}\right)$, destacaram-se com insuficiência de descrição o espectro de gotas formado pela pulverização, com ausência de informação em 84,5\% dos trabalhos avaliados, pressão de trabalho (15,5\%), concentração da calda (20,5\%), distância e posição das pontas de pulverização em relação ao alvo $(58,5 \%)$ e condições meteorológicas, com ausência média (temperatura, umidade relativa do ar e velocidade dos ventos) de 62,6\% nos artigos avaliados. Para resgatar a importância da descrição criteriosa sobre as tecnologias de aplicação de herbicidas em artigos científicos, foi elaborada uma proposta de descrição metodológica simplificada, a qual proporciona maximizar a segurança e fidelidade na reprodução das informações referentes ao controle de plantas daninhas.

Palavras-chave: tecnologia de aplicação, metodologia, padronização.

\footnotetext{
Abstract - Chemical control is a viable tool and practically essential in the management of weeds control. However, insufficient details about application methods of pesticides have been observed in most scientific publications that deal with herbicides utilization. With the objective

${ }^{1}$ Recebido para publicação em 20/03/2012 e aceito em 15/04/2012.

${ }^{2}$ Docente, Departamento de Agronomia da Universidade Estadual de Maringá, Campus Universitário - Av.: Colombo 5790, Bloco J-45, CEP 87020-900, Maringá-Paraná, Brasil, mgbalan2@uem.br <autor para correspondência>; cdgmaciel2@uem.br.

${ }^{3}$ Docente, Departamento de Agronomia, Centro de Ciências Agrárias, Universidade Estadual de Londrina, Londrina Paraná, Brasil, CEP 86047-230, abisaab@uel.com.br.

${ }^{4}$ Doutorando, Departamento de Agronomia, Centro de Ciências Agrárias, Universidade Estadual de Londrina, Londrina - Paraná, Brasil, CEP 86047-230, gmigliorini@ hotmail.com.
} 
Balan et al.

of verifying the presence or absence of basic information about herbicides application found in scientific papers, as well as to propose a criterion of minimum description needed with information that may guarantee better repeatability of methods and techniques used, it was conducted a survey in 200 papers published in national and international periodic. It was observed that minimum descriptions required about herbicides application technology have not been observed. Besides all papers showed the used application rate ( $\mathrm{L} \mathrm{ha}^{-1}$ ), outstanding with insufficiency the description of droplets spectrum formed by sprayer, with lack of information in $84.5 \%$ of evaluated works; work pressure (15.5\%); spray solution concentration (20.5\%); distance and position of nozzles in relation to the target $(58.5 \%)$ and meteorological conditions, with median absence (temperature, air humidity and winds speed) of $62.6 \%$ in evaluated papers. In order to rescue the importance of criterion description about herbicides application technologies in scientific papers, it was elaborated a simplified methodological description, that offers to maximize the security and fidelity in information's reproduced referred to weeds control.

Keywords: application technology, methodology, uniformity.

\section{Introdução}

Significativas mudanças e inovações tecnológicas, advindas do aumento da incidência de plantas daninhas de importância têm ocorrido na defesa fitossanitária nos últimos 30 anos. Nesse contexto, o manejo e controle das plantas daninhas com herbicidas têm contribuído com aproximadamente $49,0 \%$ do volume total dos produtos fitossanitários comercializados no Brasil de 1992 a 2005, contabilizando US\$ 16 bilhões (SINDAG, 2005).

Afora seu grande impacto econômico, os produtos fitossanitários são alvos de crescente preocupação em virtude de seu risco ambiental e à saúde humana (Barcellos et al., 1998; Cunha et al., 2003; Matthews, 2008; Conceição, 2008). A Agenda 21, em capítulo específico sobre produtos fitossanitários, envolve a questão dos riscos e efeitos colaterais desses produtos nos diversos âmbitos de utilização, objetivando contribuir para definição de seu emprego, inclusive das quantidades a serem aplicadas, por profissionais habilitados e treinados (CNUMAD, 1996; Barbieri, 1997). Conceição (2006) destaca comparativamente a redução expressiva da ordem de $89,11 \%$ da dose de ingrediente ativo dos herbicidas recomendados e empregados no Brasil, entre as décadas de 60 a 90 .

A utilização da tecnologia de aplicação busca a deposição da quantidade indicada de ingrediente ativo no alvo pretendido, com o máximo de eficiência e da maneira mais economicamente viável, evitando afetar o ambiente (Matthews, 2002; Matuo et al., 2006). O domínio dessa tecnologia é imprescindível para aumentar a eficiência do produto e diminuir a contaminação do aplicador e ambiente, bem como na redução dos custos nas aplicações.

Matthews (2004, 2008) evidencia diminuições expressivas nos volumes de aplicação em pulverizações terrestres de $500 \mathrm{~L}$ $\mathrm{ha}^{-1}$ para quantidades inferiores a $200 \mathrm{~L} \mathrm{ha}^{-1}$, sendo requeridos aprimoramentos nestas aplicações empregadas no campo, buscando a máxima precisão e dosagem ótima, com o mínimo de exposição para o operador. Nesse contexto, destaca que o desenvolvimento de novas pontas de pulverização proporcionando maior flexibilidade e aperfeiçoando a distribuição dos produtos fitossanitários, e assegurando redução nos volumes de pulverização sem influenciar as transferências de doses. 
Balan et al.

O espectro de gotas formadas em pontas hidráulicas é determinado pelo modelo de ponta, tamanho do orifício (vazão nominal), ângulo de descarga (projeção), pressão de trabalho e formulação do produto fitossanitário. Esses fatores, portanto, estarão devidamente relacionados à cobertura do alvo pela calda pulverizada, uma vez que, fixando-se o volume de aplicação e a área da cultura a ser coberta, dentro dos limites da deriva e do ponto de escorrimento, a cobertura poderá ser afetada pela alteração do espectro de gotas. Assim, determina-se a essencialidade da seleção apropriada das pontas de pulverização, que são responsáveis pela quantidade aplicada por área, uniformidade da cobertura obtida e pelo risco potencial de deriva (Matthews, 2004, 2008; Van de Zande et al., 2008; Lan et al., 2008).

O sistema internacional de classificação do espectro de gotas formadas e de pontas de pulverização baseia-se em dois componentes: distribuição do tamanho de gotas e risco de deriva (Miller et al., 2002). Essa classificação está apresentada, inclusive, com os padrões de cores das pontas de pulverização com a norma ASAE S-572 da ASABE (2004). Portanto, o grande desafio da pesquisa neste sentido é a obtenção da cobertura ideal do alvo com distribuição uniforme das gotas produzidas.

Por um lado as gotas muito grandes podem apresentar problemas de cobertura insuficiente do alvo e desuniformidade de distribuição, assim como excesso de massa que interfere em sua aderência ao alvo e na perda para o solo. Do outro, gotas pequenas podem evaporar em condições de baixa umidade relativa ou serem levadas por corrente de ar, intensificando e provocando o fenômeno da deriva, com aumento do risco de contaminação ambiental (Ferreira, 2003; Cunha et al., 2003; Zhu et al., 2004; Jamar et al., 2010; Wolf \& Daggupati, 2009; Fritz et al., 2007). Velloso \& Souza (1996) relataram que temperaturas do ar acima de $30^{\circ} \mathrm{C}$ e umidade relativa abaixo de $70 \%$ favorecem a ocorrência da deriva, devendo ser monitoradas as condições ambientais na ocasião da aplicação. Segundo Ruedell (1995, 1999, 2002), poucas pesquisas levam em consideração as condições ambientais nas aplicações de produtos fitossanitários. Fritz (2006) e Yu et al. (2009) evidenciaram a importância e influência das condições meteorológicas na performance das pulverizações.

Matthews (2002) menciona que cada ponta de pulverização possui característica própria de distribuição volumétrica, sendo esta específica para condição de altura da ponta em relação ao alvo e espaçamento entre as pontas na barra de pulverização. No Brasil, o uso do espaçamento entre bicos de $50 \mathrm{~cm}$ é o mais frequente, não levando por vezes em consideração a altura da ponta em relação ao alvo e espaçamento entre pontas. Portanto, conhecendo-se a ponta e o jato emitido pode-se buscar melhorar a eficiência no controle do alvo modificando a relação entre esse espaçamento e altura mínima da barra (Christofoletti, 2000; Peressin \& Perecin, 2003; Cunha \& Ruas, 2006).

Apesar de todas essas considerações e esforços para uma prática mais segura e eficiente de aplicação de produtos fitossanitários, observa-se que ainda é dada muita importância ao produto fitossanitário e pouca à técnica de aplicação (Hislop, 1991; Cunha et al., 2003). Para Ramos (2001) o desconhecimento dos conceitos de aplicação de produtos fitossanitários é evidente na literatura científica, exemplificando que, com frequência, o volume de calda aplicado é considerado como parâmetro suficiente para caracterizar e proporcionar a repetibilidade de uma determinada aplicação.

Mesmo na importante missão em buscar notoriedade científica por meio de publicações em periódicos de grande impacto na comunidade científica, detalhes insuficientes sobre os métodos de aplicação de produtos fitossanitários têm sido reportados na maioria 
Balan et al.

dessas publicações. Segundo Matthews (2004), a maioria dos trabalhos científicos não traz informações descritivas suficientes em detrimento aos métodos de aplicação utilizados. Afora o volume de água utilizada para diluição, presente em todas as publicações, o autor destaca falta de informações sobre o os tipos de pontas utilizadas, ângulo e categoria do espectro de gotas formada, concentração da calda pulverizada, pressão de trabalho e sua manutenção, posição das pontas em relação à cultura, assim como das condições meteorológicas no momento da aplicação.

Essa realidade pode reduzir a credibilidade da informação, uma vez que prejudica a distinção se o que resultou em um tratamento fitossanitário ineficiente foi determinado pelo produto aplicado ou pela técnica inadequada de aplicação. Dentro deste contexto, o objetivo do presente trabalho foi verificar a presença ou ausência de descrições metodológicas básicas sobre aplicação de herbicidas utilizados em artigos científicos, bem como propor uma descrição mínima necessária dessas informações, de forma a oferecer subsídios técnicos para maximizar a segurança e a fidelidade na reprodução das informações.

\section{Material e Métodos}

Com base no trabalho de Matthews (2004), considerando sua afirmação sobre a insuficiência de informações metodológicas básicas sobre a técnica de aplicação de produtos fitossanitários, foi realizado levantamento em base bibliográfica disponível para consulta no portal de periódicos da CAPES (2006), periódicos e bases bibliográficas de livre acesso disponíveis online (Internet); periódicos, jornais e revistas científicas disponíveis nas bibliotecas da Universidade Estadual de Londrina (UEL), Universidade Estadual de Maringá (UEM), Escola Superior de agricultura "Luiz de
Queiroz" (ESALQ/USP) e Faculdade Integrado de Campo Mourão. Foram selecionados 200 artigos que abordam a aplicação de herbicidas, sendo 100 destes publicados em periódicos nacionais e 100 em internacionais. Os trabalhos selecionados foram aqueles com ano de publicação superior a 1990.

Como critério os trabalhos consultados foram, preferencialmente, aqueles inseridos na lista de Qualis da CAPES (Coordenação de Aperfeiçoamento de Pessoal de Nível Superior) referente ao triênio 2004/2006. O Qualis da CAPES classifica os veículos de divulgação (periódicos) da produção intelectual (bibliográfica) dos programas de pósgraduação stricto sensu, assim como também é utilizado pela própria Capes para o processo de avaliação de seus programas pós-graduação.

Foram verificadas a presença ou ausência das seguintes informações na descrição metodológica dos trabalhos científicos avaliados: a) ponta de pulverização: descrição (tipo/modelo); ângulo de descarga (projeção) e espectro de gotas formado; b) pressão de trabalho: descrição da pressão utilizada e sua manutenção; c) taxa de aplicação: descrição do volume da calda aplicada por área; d) concentração da calda aplicada: descrição da dosagem do ingrediente ativo utilizado por volume de aplicação; e) temperatura do ar, f) umidade relativa do ar, e g) velocidade dos ventos: informação no momento da aplicação.

Para as bases bibliográficas avaliadas, nacionais e internacionais, os resultados das informações foram computados como presentes (Sim) ou ausentes (Não) sendo dispostos em gráficos em porcentagem, para posterior visualização do panorama geral, seguida de descrição e análise. Optou-se pela não identificação individual da autoria dos trabalhos avaliados, por não se tratar do objetivo do presente trabalho, sendo apenas referenciada quantitativamente a origem das publicações (Tabela 1). 
Balan et al.

Tabela 1. Base do referencial bibliográfico consultado e sua distribuição quantitativa.

\begin{tabular}{|c|c|c|}
\hline \multirow{2}{*}{ FONTE } & \multicolumn{2}{|c|}{ Número de publicações avaliadas } \\
\hline & Nacionais & Internacionais \\
\hline Agronomy Journal & - & 17 \\
\hline Arquivos do Instituto Biológico & 5 & - \\
\hline Aspects of Applied Biology & - & 9 \\
\hline Biosystems Engineering & - & 7 \\
\hline Bragantia & 7 & - \\
\hline Ciência e Agrotecnologia & 5 & - \\
\hline Ciência Rural & 13 & - \\
\hline Crop Protection & - & 27 \\
\hline Engenharia Agrícola & 8 & - \\
\hline Horticultura Brasileira & 6 & - \\
\hline Journal of Agricultural Engineering Research & - & 5 \\
\hline Journal of Environmental Quality & - & 5 \\
\hline Journal of Environmental Science and Health & - & 5 \\
\hline Pesquisa Agropecuária Brasileira & - & 7 \\
\hline Planta Daninha & 27 & - \\
\hline Revista Brasileira de Agrociência & 5 & - \\
\hline Revista Brasileira de Engenharia Agrícola Ambiental & 9 & - \\
\hline Revista Brasileira de Fruticultura & 7 & - \\
\hline Revista Brasileira de Herbicidas & 8 & - \\
\hline Transactions of the American Society of Engineering & - & 6 \\
\hline Weed Science & - & 6 \\
\hline Weed Technology & - & 6 \\
\hline TOTAL & 100 & 100 \\
\hline
\end{tabular}

\section{Resultados e Discussão}

Das informações referentes as pontas de pulverização, o fator mais evidente e também preocupante é a falta de informação do espectro de gotas formado nos trabalhos de pesquisa, visto que, dos 200 trabalhos avaliados, 84,5\% não disponibilizaram esse dado (Figura 1). Apesar do grande desenvolvimento da tecnologia para as pontas de pulverização e as tentativas de normatização das mesmas por meio de esforços internacionais, buscando aplicações mais precisas, econômicas, seguras ao ambiente/saúde humana e focadas na redução do potencial de deriva, a utilização correta das tecnologias geradas para aplicações continua sendo apenas uma tendência (Matthews, 2004). 
Balan et al.

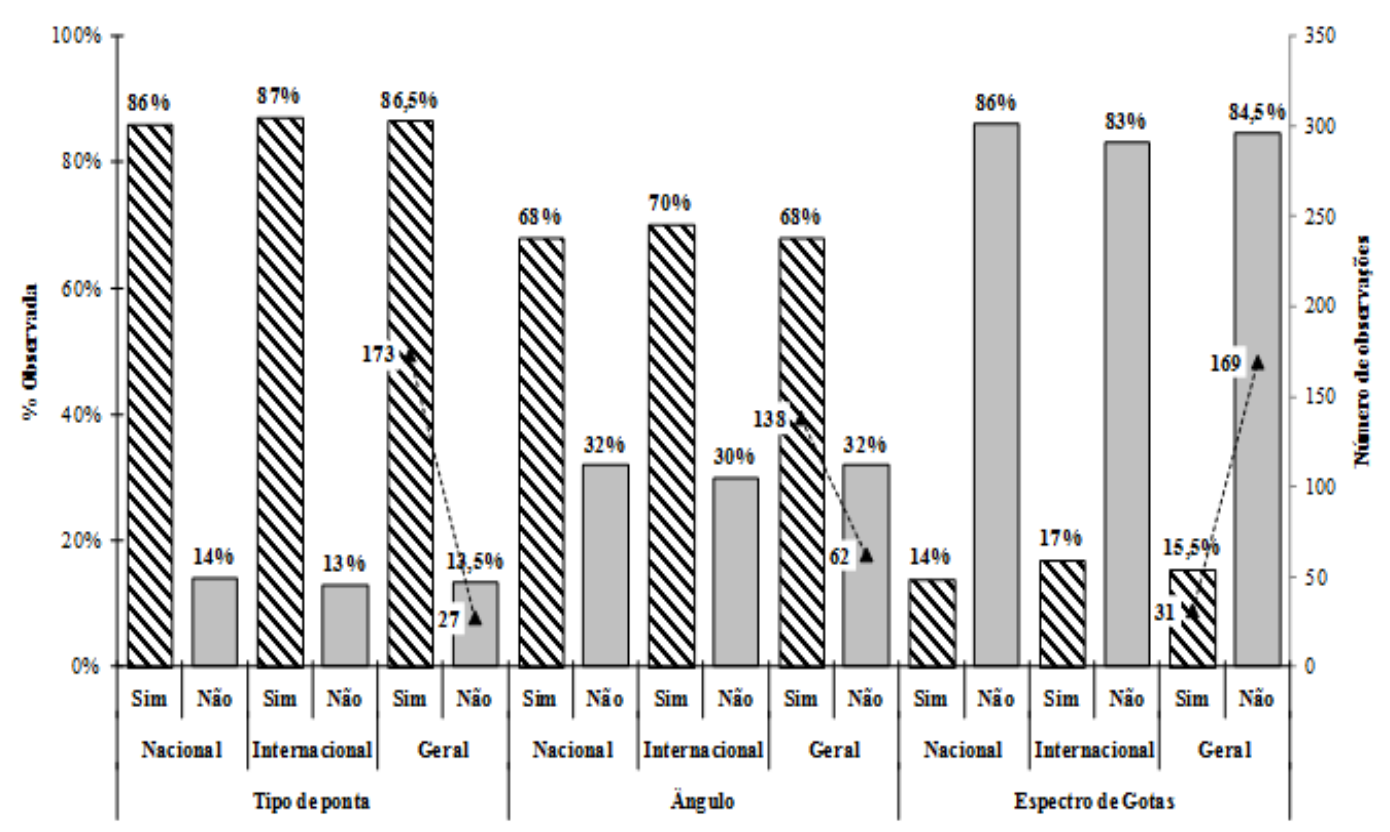

Figura 1. Distribuição porcentual e numérica de informações sobre o tipo de ponta de pulverização utilizada, ângulo de projeção e espectro de gotas formadas em artigos científicos (nacionais e internacionais) sobre aplicação de herbicidas.

Com relação ao tipo de ponta utilizada nas aplicações, apenas $13,5 \%$ dos trabalhos científicos, veiculados nacionalmente e internacionalmente, não apresentaram essa informação. Para o ângulo de projeção das pontas nas aplicações, nota-se assim como para o espectro de gotas, que esta característica pode não ter significado relevante para os pesquisadores, talvez pela de possibilidade de consulta aos manuais de fabricantes e/ou de trabalhos específicos. O espectro de gotas formadas e a disposição correta desse em relação ao alvo pretendido são importantes e deveriam ser consideradas na descrição das metodologias dos artigos científicos, já que cada tipo de ponta de pulverização possui uma característica própria (Matthews, 2002).

Quanto à pressão de trabalho, que é o princípio básico da pulverização hidráulica, onde se fundamenta a formação e manutenção da distribuição das gotas, não foi metodologicamente descrita em 15,5\% (31) dos artigos avaliados (Figura 2). Por ser uma variável em diferentes equipamentos, a falta da informação sobre pressão de trabalho pode inviabilizar a reprodução da aplicação técnica de herbicidas. Desta forma, para essa característica deve-se atenção especial, onde deveria haver menção em todos os trabalhos que tratam de aplicação de produtos fitossanitários. Em levantamento nacional de inspeção de pulverizadores, onde foram avaliados 200 pulverizadores de barra, verificou-se que todos apresentaram algum tipo de falha e relatam que problemas com manômetros $(92,3 \%)$, pontas de pulverização $(80,5 \%)$ e vazamentos (54\%) lideraram o ranking das desconformidades (Antuniassi \& Gandolfo, 2002).

Com relação a dosagem do herbicida (concentração da calda), em 20,5\% (42) dos trabalhos avaliados essa informação não apresentava-se disponível (Figura 3). Como há a tendência, e têm sido praticadas pulverizações terrestres de herbicidas em taxas (volumes) de aplicação inferiores a $200 \mathrm{~L} \mathrm{ha}^{-1}$, 
Balan et al.

e levando ainda em consideração a altas, se a técnica de aplicação não for recomendação de doses de herbicidas por apropriada o seu efeito pode ser reduzido ou unidade de área (ha), a indicação da potencializado em detrimento às transferências concentração do ingrediente ativo (dose) por inadequadas de doses resultando, em ambos os volume de aplicação é importante e deve ser citada. Apesar das doses recomendadas serem casos, em perdas de eficiência ou lucratividade (Matthews, 2008).

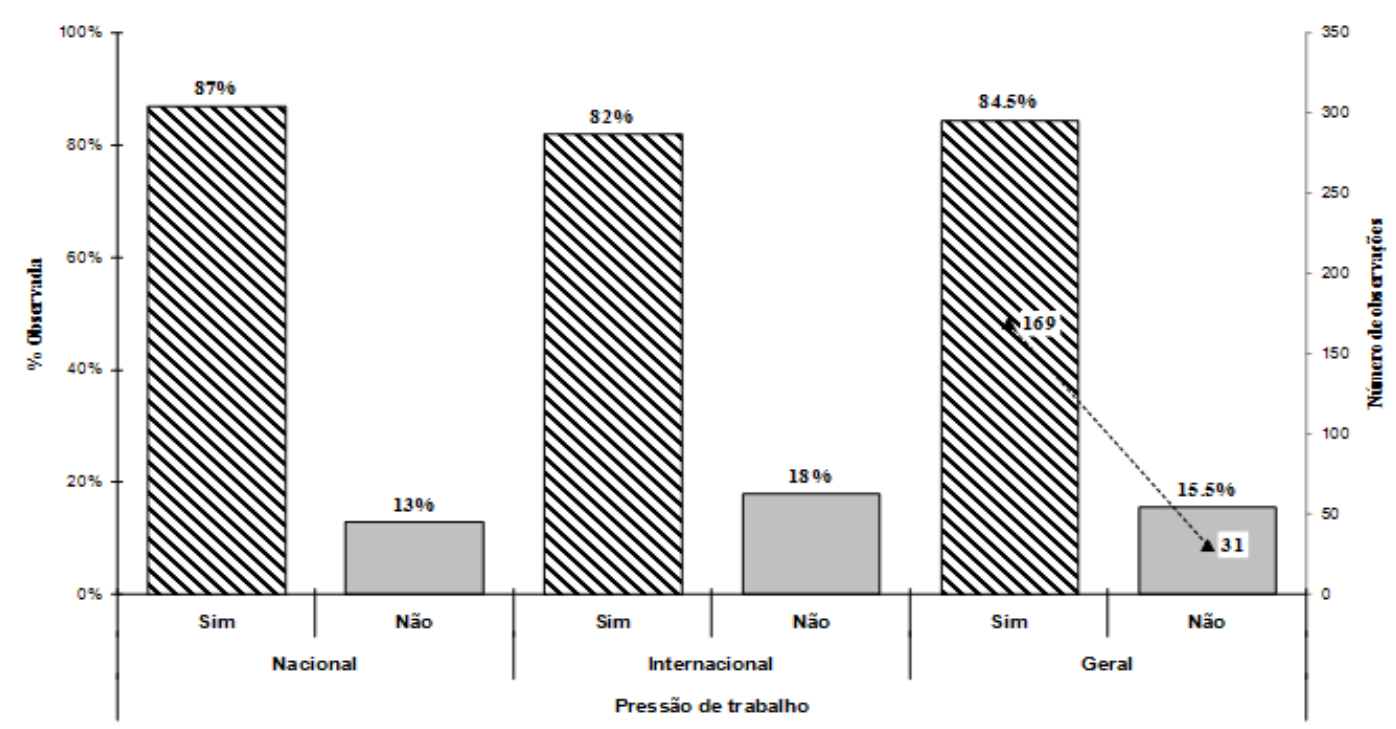

Figura 2. Distribuição porcentual e numérica de informações sobre a pressão de trabalho em artigos científicos (nacionais e internacionais) sobre aplicação de herbicidas.

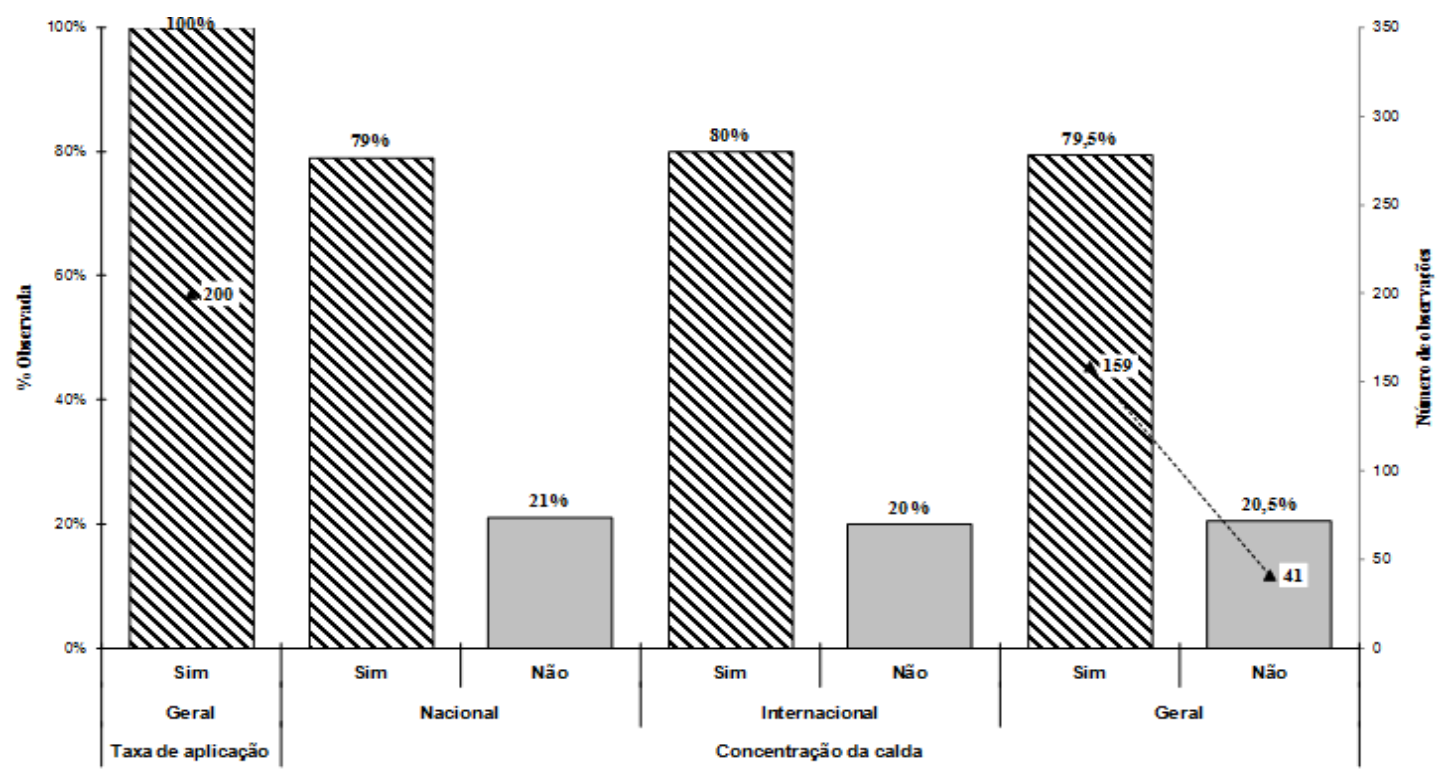

Figura 3. Distribuição porcentual e numérica de informações sobre a taxa de aplicação e concentração da calda de pulverização praticada em artigos científicos (nacionais e internacionais) sobre aplicação de herbicidas. 
Balan et al.

Para a distância e posição das pontas em relação ao alvo, também verificou-se um item pouco apreciado e de baixa relevância nas publicações analisadas, exceto em trabalhos específicos com tecnologia de aplicação (Figura 4). Essa informação não esteve presente em 58,5\% (117) dos trabalhos, tornando esses valores preocupantes, pois, diversas são as possibilidades de espaçamento entre as pontas e a relação desses espaçamentos com a altura (distância) ao alvo. Estes ajustes na aplicação variam em função do modelo da ponta, da pressão de trabalho, do tipo de calda, do ângulo do jato emitido, assim como da topografia do terreno, da densidade foliar e/ou arquitetura da cultura, podendo alterar expressivamente $\mathrm{o}$ resultado da pulverização, resultando em coeficientes de variação excessivos e, consequente, deposição desuniforme da aplicação. A aplicação desuniforme pode prejudicar a eficiência dos herbicidas havendo, em muitos casos, a necessidade de repasse para o efetivo controle pretendido (Perecin et al., 1998; Peressin \& Perecin, 2003).

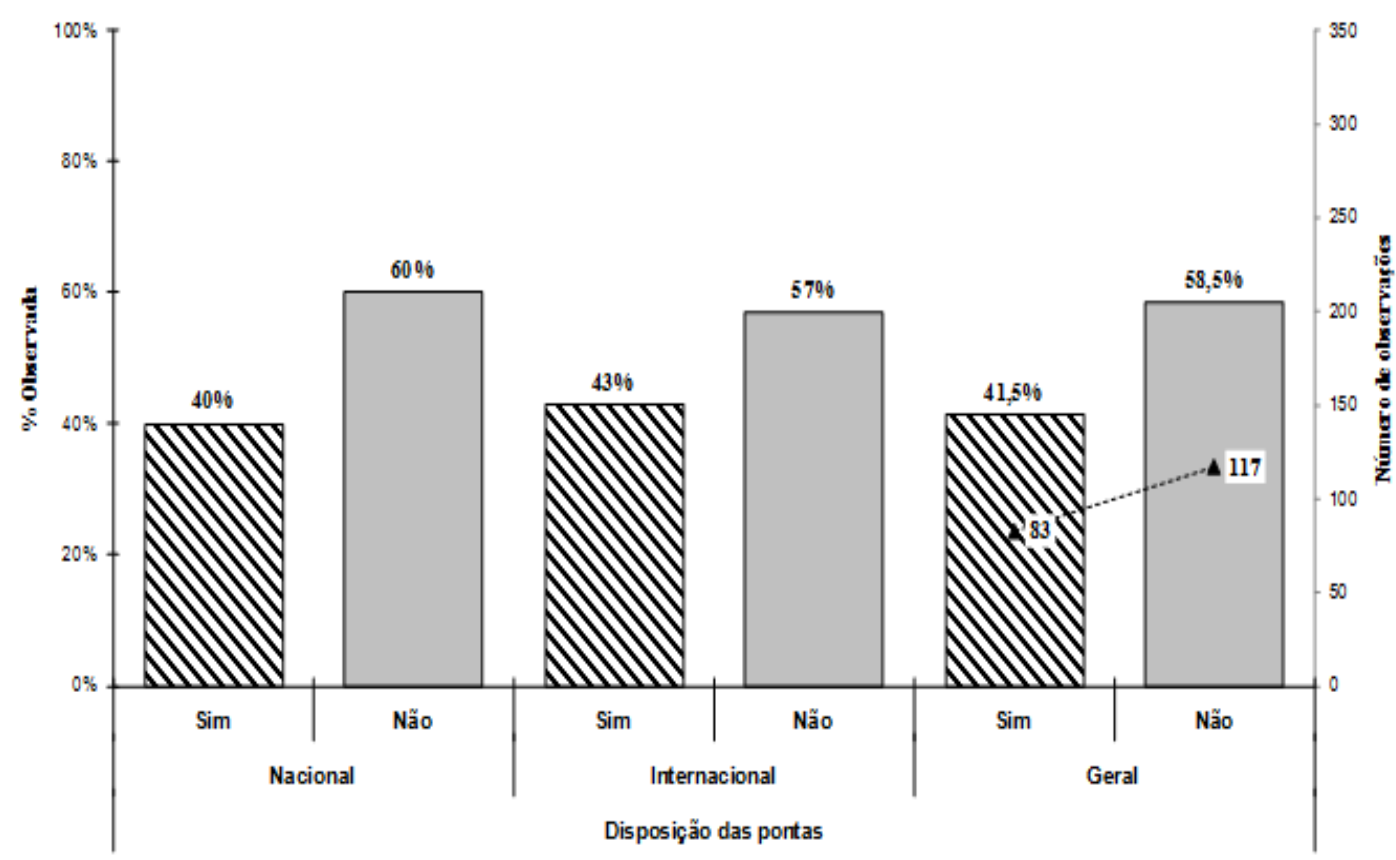

Figura 4. Distribuição porcentual e numérica de informações sobre a distância e posição da ponta em relação ao alvo em artigos científicos (nacionais e internacionais) sobre aplicação de herbicidas.

Quando avaliados os itens temperatura, umidade relativa do ar e velocidade dos ventos (Figura 5), cabe o alerta da respectiva inobservância de $60,5 \%, 61 \%$ e $66,5 \%$ nos trabalhos avaliados, estando de acordo com o relatos de Ruedell (1995, 1999, 2002) sobre a falta de preocupação das pesquisas científicas com as condições do ambiente nas pulverizações. Muitos são os fatores que podem contribuir para o sucesso ou fracasso da aplicação de herbicidas, entre esses a observação das condições climáticas é fundamental para iniciar ou paralizar uma aplicação (Freitas \& Ferreira, 2007).

Nesse sentido, é importante ressaltar que em condições climáticas adversas, com temperaturas elevadas, baixa umidade relativa do ar e alta velocidade de vento aumentam-se 
Balan et al.

o risco de contaminação ambiental por deriva. Desta maneira, o monitoramento climático além de ser efetuado, deve ser interpretado de forma mais compreensiva possível. A falta de atenção com a umidade relativa do ar, por estar favoravelmente acima do limite crítico de monitoramento de $70 \%$, como proposto por Velloso \& Costa (1996) e Ruedell (2002), pode favorecer a ocorrência do orvalho sobre as plantas, influenciando na diluição do herbicida aplicado e até mesmo provocando seu escorrimento.

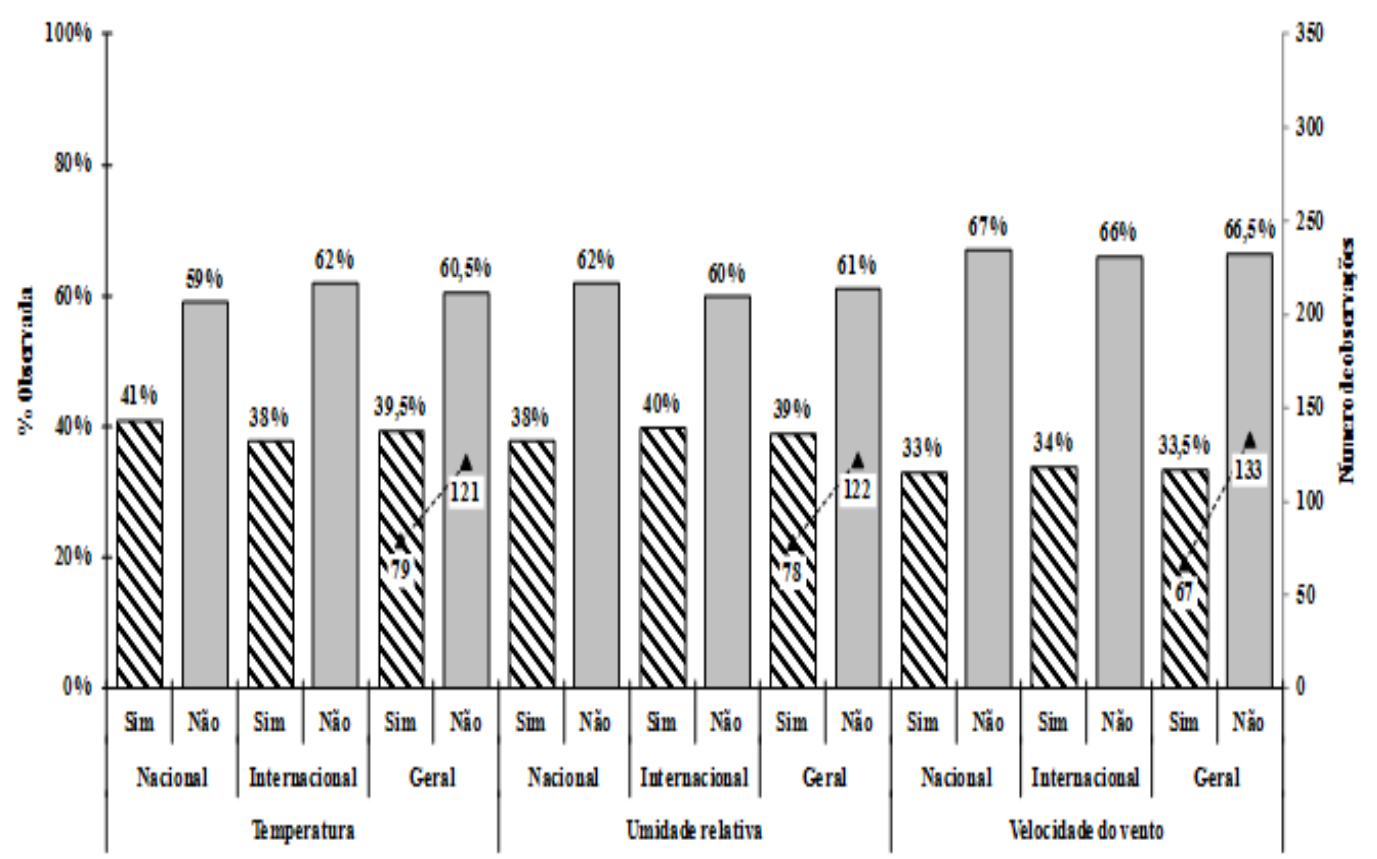

Figura 5. Distribuição porcentual e numérica de informações sobre as condições meteorológicas em artigos científicos (nacionais e internacionais) sobre aplicação de herbicidas.

Em função dos resultados observados no presente trabalho, propõe-se como sugestão uma descrição metodológica simplificada, baseada em um checklist de informações básicas que seriam necessárias constar nos artigos técnicos/científicos envolvidos com a aplicação de herbicidas. Na Tabela 2 encontram-se os iténs considerados na referida proposta que visam auxiliar na identificação, reconhecimento e seleção das informações mínimas necessárias para repetibilidade adequada de trabalhos que envolvam procedimentos da aplicação de herbicidas. Nessa proposta, alguns itens apenas reforçam e destacam a importância daquelas informações comumente descritas, conforme os resultados obtidos nesse trabalho.

Com essas informações descritas na Tabela 2 as pesquisas envolvendo aplicação de herbicidas poderão reportar as mesmas metodologias de forma padronizada, assegurando a obtenção aprimorada de resultados, sendo possível a reprodução fiel pela tecnologia de aplicação utilizada. Assim, essa classe de produção científica será beneficiada e subsidiará uma evolução na reprodução das informações para profissionais e difusores tecnológicos, que pelo princípio de extensão rural, difundirão essas informações à campo, beneficiando todos os mais diferentes segmentos da cadeia produtiva agrícola. 
Balan et al.

Tabela 2. Proposta de itens que devem ser mencionados na descrição metodológica de artigos técnicos/científicos que tratem da aplicação de herbicidas.

\begin{tabular}{|c|c|c|c|c|}
\hline \multicolumn{5}{|c|}{ Descrição do produto } \\
\hline Classe toxicológica & Formulação & $\begin{array}{c}\text { Mecanismo de ação; } \\
\text { seletividade e } \\
\text { fitotoxicidade } \\
\end{array}$ & Adjuvante (s) & $\begin{array}{l}\text { Dose recomendada } x \\
\text { Dose utilizada }\end{array}$ \\
\hline $\begin{array}{l}\text { Descrever a classe } \\
\text { toxicológica do } \\
\text { produto a ser aplicado }\end{array}$ & $\begin{array}{l}\text { Tipo de formulação } \\
\text { do produto aplicado }\end{array}$ & $\begin{array}{c}\text { Descrição se sistêmico } \\
\text { ou de contato; } \\
\text { seletividade e/ou } \\
\text { condições de } \\
\text { fitotoxicidade }\end{array}$ & $\begin{array}{c}\text { Descrição, } \\
\text { concentração e } \\
\text { caracterização } \\
\text { (quando utilizado) }\end{array}$ & $\begin{array}{c}\text { Indicar a dose recomendada } \\
\text { pelo fabricante (registrada). } \\
\text { Indicar a dose utilizada na } \\
\text { aplicação }\end{array}$ \\
\hline \multicolumn{5}{|c|}{ Aplicação } \\
\hline $\begin{array}{l}\text { Modalidade da } \\
\text { aplicação }\end{array}$ & $\begin{array}{c}\text { Taxa de aplicação } \\
\text { recomendada } \\
\left(\mathrm{L} \mathrm{ha}^{-1}\right)\end{array}$ & $\begin{array}{c}\text { Taxa de aplicação } \\
\text { utilizada } \\
\left(\mathrm{L} \mathrm{ha}^{-1}\right)\end{array}$ & $\begin{array}{l}\text { Pressão de } \\
\text { trabalho }\end{array}$ & Agente diluente \\
\hline $\begin{array}{l}\text { Pré-semeadura; pré- } \\
\text { emergência; pós- } \\
\text { emergência; pós- } \\
\text { emergência dirigida }\end{array}$ & $\begin{array}{l}\text { Informar o volume } \\
\text { de calda } \\
\text { recomendado pelo } \\
\text { fabricante }\end{array}$ & $\begin{array}{l}\text { Informar o volume de } \\
\text { calda efetivamente } \\
\text { aplicada }\end{array}$ & $\begin{array}{l}\text { Identificar a pressão } \\
\text { de trabalho utilizada } \\
\qquad(\mathrm{kPa})\end{array}$ & $\begin{array}{c}\text { Se disponível e possível, } \\
\text { descrever a qualidade da } \\
\text { água utilizada (dureza e } \\
\mathrm{pH} \text { ) }\end{array}$ \\
\hline \multicolumn{5}{|c|}{ Aplicações realizadas } \\
\hline Data e horário & $\begin{array}{l}\text { Número de } \\
\text { aplicações }\end{array}$ & $\begin{array}{l}\text { Intervalo entre } \\
\text { aplicações }\end{array}$ & Descrição do alvo & $\begin{array}{c}\text { Descrição do cultivo } \\
\text { protegido }\end{array}$ \\
\hline $\begin{array}{c}\text { Informar a data e o } \\
\text { horário da execução de } \\
\text { cada aplicação } \\
\text { realizada } \\
\end{array}$ & $\begin{array}{l}\text { Descrever o número } \\
\text { de aplicações } \\
\text { realizadas }\end{array}$ & $\begin{array}{l}\text { Informar o intervalo } \\
\text { em dias entre as } \\
\text { aplicações realizadas }\end{array}$ & $\begin{array}{l}\text { Descrever o estádio } \\
\text { fenológico, } \\
\text { densidade, espécie e } \\
\text { altura do alvo }\end{array}$ & $\begin{array}{l}\text { Descrever estádio } \\
\text { fenológico e altura das } \\
\text { plantas da cultura }\end{array}$ \\
\hline \multicolumn{5}{|c|}{ Ponta de pulverização } \\
\hline Modelo & $\begin{array}{c}\text { Ângulo de } \\
\text { descarga } \\
\text { (projeção) }\end{array}$ & $\begin{array}{l}\text { Vazão } \\
\left(\mathbf{L} \text { min }^{-1}\right)\end{array}$ & $\begin{array}{l}\text { Espectro de gotas } \\
\text { formado* }\end{array}$ & $\begin{array}{c}\text { Diâmetro mediano } \\
\text { volumétrico (DMV) }\end{array}$ \\
\hline $\begin{array}{l}\text { Informar o modelo da } \\
\text { ponta (fabricante) }\end{array}$ & $\begin{array}{c}\text { Informar se } \\
\text { disponível (graus) }\end{array}$ & $\begin{array}{c}\text { Informar a vazão } \\
\text { nominal da ponta de } \\
\text { pulverização }\end{array}$ & $\begin{array}{c}\text { Categoria do } \\
\text { espectro de gotas } \\
\text { formado na pressão } \\
\text { de trabalho utilizada }\end{array}$ & Informar se disponível \\
\hline \multicolumn{5}{|c|}{ Equipamento } \\
\hline Modelo & Tipo de barra & $\begin{array}{c}\text { Espaçamento entre } \\
\text { bicos }\end{array}$ & Ângulo dos bicos & $\begin{array}{c}\text { Altura de operação da } \\
\text { barra/ assistência de ar }\end{array}$ \\
\hline $\begin{array}{l}\text { Descrever o tipo de } \\
\text { equipamento, } \\
\text { capacidade de } \\
\text { operação e manutenção } \\
\text { da pressão de trabalho }\end{array}$ & $\begin{array}{l}\text { Comprimento e } \\
\text { descrição de sua } \\
\text { montagem no } \\
\text { equipamento }\end{array}$ & $\begin{array}{l}\text { Espaçamento entre os } \\
\text { bicos de pulverização } \\
\text { utilizados na aplicação }\end{array}$ & $\begin{array}{c}\text { Se utilizado } \\
\text { descrever a } \\
\text { angulação dos bicos } \\
\text { em relação à } \\
\text { vertical. }\end{array}$ & $\begin{array}{c}\text { Informar a distância } \\
\text { utilizada entre as pontas de } \\
\text { pulverização e o alvo } \\
\text { pretendido. Informar o tipo } \\
\text { de assistência de ar } \\
\text { utilizada }\end{array}$ \\
\hline \multicolumn{5}{|c|}{ Condições meteorológicas } \\
\hline Temperatura & Umidade relativa & Velocidade do vento & $\begin{array}{c}\text { Umidade do solo e } \\
\text { Nebulosidade }\end{array}$ & Precipitação \\
\hline $\begin{array}{l}\text { Informar a temperatura } \\
\text { no momento da } \\
\text { aplicação. }\end{array}$ & $\begin{array}{l}\text { Informar a umidade } \\
\text { relativa do ar no } \\
\text { momento da } \\
\text { aplicação }\end{array}$ & $\begin{array}{l}\text { Informar a velocidade } \\
\text { do vento no momento e } \\
\text { durante a aplicação }\end{array}$ & $\begin{array}{l}\text { Identificar as } \\
\text { condições de } \\
\text { umidade do solo e } \\
\text { nebulosidade no } \\
\text { momento da } \\
\text { aplicação }\end{array}$ & $\begin{array}{l}\text { Informar se ocorrer (antes, } \\
\text { durante e após a aplicação) }\end{array}$ \\
\hline
\end{tabular}

\section{Segurança}

Acrescentar informações sobre o uso de equipamento de proteção individual (EPI) por ocasião do preparo da calda e aplicação do produto fitossanitário para reforçar sua necessidade para a segurança do operador.

* A classificação do espectro de gotas internacional considerada a categoria de gotas formadas como muito fina, fina, média, grossa, muito grossa e extremamente grossa. Essa classificação está apresentada, inclusive com os padrões de cores das pontas de pulverização com a norma ASAE S572 da ASABE (2004). 
Balan et al.

\section{Conclusão}

Não foram observadas descrições satisfatórias para repetibilidade de trabalhos científicos envolvendo a aplicação de herbicidas. As principais ausências de informações básicas foram referente às características das pontas de pulverização, concentração da calda pulverizada, pressão de trabalho, posição das pontas em relação à cultura/planta alvo, assim como das condições meteorológicas no momento da aplicação.

A proposta de descrição metodológica simplificada das tecnologias de aplicação de herbicidas em artigos científicos proporciona maximizar a segurança e fidelidade na reprodução das informações, referentes ao controle de plantas daninhas.

\section{Referências}

AMERICAN SOCIETY OF AGRICULTURAL AND BIOLOGICAL ENGINEERS -ASABE. Spray Nozzle Classification by Droplet Spectra. St. Joseph: ASAE, Norma S-572, p.437-440, 2004.

ANTUNIASSI, U.R.; GANDOLFO, M.A. Velhos e sem manutenção. Cultivar Máquinas, v.2, n.14, p.25-27, 2002.

BARBIERI, J.C. Desenvolvimento e Meio Ambiente. Estratégias de mudanças da Agenda 21. Petrópolis: Editora Vozes, 6 a . Ed., 1997, 159p.

BARCELLOS, L.C.; CARVALHO, Y.C.; SILVA, A.L. Estudo sobre a penetração de gotas de pulverização no dossel da cultura da soja [Glycine max (L.) Merrill]. Engenharia Agrícola, v.6, n.2, p.81-94, 1998.

CHRISTOFOLETTI, P.J. A importância da distribuição de uma ponta de pulverização. São Paulo: TeeJet South América, 2000. 7p. (Boletim Técnico BT-10/2000).
CONCEIÇÃO, M.Z. Defesa Vegetal: Legislação, Normas e Produtos Fitossanitários. In: $O$ que os engenheiros agrônomos devem saber para orientar o uso de produtos fitossanitários. 3. ed. rev. ampl., Viçosa: UFV/DFP, p.1-61, 2008.

CONCEIÇÃO, M.Z. Proteção de plantas. In: ABEAS - Curso de proteção de plantas. (Módulo 1 - 1.1). Brasília: ABEAS; Viçosa: UFV, 2006. 56p.

Conferência das Nações Unidas sobre Meio Ambiente e Desenvolvimento (CNUMAD), 1992. Agenda 21. Brasília: Senado Federal, 1996. 591p.

CUNHA, J.P.A.R. et al. Avaliação de estratégias para redução da deriva de agrotóxicos em pulverizações hidráulicas.

Planta Daninha, v.21, n.2, p.325-332, 2003.

CUNHA, J.P.A.R.; RUAS, R.A.A. Uniformidade de distribuição volumétrica de pontas de pulverização de jato plano duplo com indução de ar. Pesquisa Agropecuária Tropical, v.36, n.1, p.61-66, 2006.

FERREIRA, F.B. Resposta do arroz irrigado (Oryza sativa) a subdoses de glyphosate e butroxydim simulando efeito de deriva. 2003. 52p. Dissertação (Mestrado em Fitossanidade) - Universidade Federal de Pelotas, Pelotas, 2003.

FREITAS, F.C.L; FERREIRA, L.R. Tecnologia para aplicação de herbicidas. In: FREITAS, F.C.L et al. (ed.) SIMPÓSIO SOBRE MANEJO DE PLANTAS DANINHAS NO SEMI-ÁRICO, 1., 2007 Anais... Mossoró: UFERSA, 2007. p.225-252.

FRITZ, B.K.; HOFFMANN, W.C.; MARTIN, D.E.; THOMSON, S.J. Aerial application methods for increasing spray deposition on wheat heads. Applied Engineering in Agriculture, v.23, n.6, p.709-715, 2007.

FRITZ, B.K. Meteorological effects on deposition and drift of aerially applied sprays. 
Balan et al.

Transactions of the ASABE, v.49, n.5, p.1295-1301, 2006.

HISLOP, E.C. Air assisted crop spraying: an introductory review. In: LAVERS, A.; HERINGTON, P.; SOUTHCOMBE, E.S.E. (ed.). Air-assisted spraying in crop protection. Swansea: British Crop Protection Council, 1991. p.3-14 (BCPC Monograph, 46).

JAMAR, L.; MOSTADE, O.; HUYGHEABAERT, B.; PIGEON, O. Comparative performance of recycling tunnel and conventional sprayers using standard and drift-mitigating nozzles in dwarf apple orchards. Crop Protection, v.29, n.6, p.561566, 2010.

LAN, Y. et al. Spray drift mitigation with spray mix adjuvants. Applied Engineering in Agriculture, v.24, n.1, p.5-10, 2008.

MATTHEWS, G.A. Developments in application technology. Environmentalist, v.28, n.1, p.19-24, 2008.

MATTHEWS, G.A. How was the pesticide applied? Crop Protection, v.23, n.7, p.651653, 2004.

MATTHEWS, G.A. The application of chemicals for plant disease control. In: WALLER, J.M.; LENNÉ, J.M.; WALLER, S.J. (ed.). Plant pathologist's pocketbook. London: CAB, p.345-53, 2002.

MATUO, T. et al. Tecnologia de aplicação e equipamentos. In: ABEAS - Curso de proteção de plantas. (Módulo 2 - 2.1;2.2). Brasília: ABEAS; Viçosa: UFV, 2006. 100p.

MILLER, P.C.H; BUTLER-ELLIS, M.C.; GILBERT, A.J. Extending the International BCPC spray classification scheme. Aspects of Applied Biology, v.66, n.3, p.17-24, 2002.

PERECIN, D. et al. Padrões de distribuição de líquidos obtidos com bicos TF-VS4, TJ6011006 e TQ 15006 em mesa de prova. Pesquisa Agropecuária Brasileira, v.33, n.2, p.175-182, 1998.
PERESSIN, V.A.; PERECIN, D. Avaliação do padrão de distribuição de bicos para aplicação de herbicidas: efeitos da altura do alvo nos padrões de distribuição. Bragantia, v.62, n.3, p.477-497, 2003.

QUALIS. Classificação de periódicos, anais, revistas e jornais. Disponível em: $<$ http://www.qualis.capes.gov.br/webqualis > Acesso em 20 dez. 2006.

RAMOS, H.H. Perdas ligadas à má aplicação de agrotóxicos. In: Simpósio Internacional de Tecnologia de Aplicação de Agrotóxicos: Eficiência, Economia e Preservação da Saúde Humana e do Ambiente, 2, 2001. Disponível em: <www.iac.br/cea/Sintag/AHamilton.PDF> em 14/11/2002. Acesso em: 23 dez. 2008.

RUEDELL, J. Tendências e estratégias na tecnologia de aplicação de herbicidas. In: CONGRESSO BRASILEIRO DE CIÊNCIA DAS PLANTAS DANINHAS, 20., 1995, Florianópolis. Palestras... Florianópolis: SBHED/UFSC, p.67-69, 1995.

RUEDELL, J. Controle de plantas daninhas. In: MATZENBACHER, R.G. A cultura da aveia no sistema de plantio direto. Cruz Alta: FUNDACEP FECOTRIGO/SENAR, p.85-119, 1999.

RUEDELL, J. Tecnologia de aplicação de defensivos. Plantio Direto, v.19, n.6, p.1-11, 2002.

SINDICATO NACIONAL DA INDÚSTRIA DE PRODUTOS PARA A DEFESA AGRÍCOLA - SINDAG, ANDEF, 2005. Disponível em: <http://www.sindag.com.br/html/ estat_dezembro.html>. Acesso em 23 dez. 2008.

VAN DE ZANDE, J.C. et al. Spray techniques: how to optimise spray deposition and minimise spray drift. Environmentalist, v.28, n.1, p.9-17, 2008. 
Balan et al.

VELLOSO, J.A.R.O; SOUZA, R.O. Tecnologia de aplicação de defensivos agrícolas com pulverizador de barra. In: GUEDES, J.C. et al. (ed.) Tecnologia e segurança na aplicação de produtos fítossanitários. Santa Maria: Universidade Federal de Santa Maria, Departamento de Defesa Fitossanitária, Sociedade de Agronomia de Santa Maria, p.31-60, 1996.

YU, Y. et al. Evaporation and coverage area of pesticide droplets on hairy and waxy leaves. Biosystems Engineering, v.104, n.3, p.324334, 2009.

WOLF, R.E.; DAGGUPATI, N.P. Nozzle type effect on soybean canopy penetration. Applied Engineering in Agriculture, v.25, n.1, p.5-10, 2009.

ZHU, H. et al. Spray penetration into peanut canopies with hydraulic nozzle tips. Biosystems Engineering, v.87, n.3, p.275283, 2004. 